\title{
Advisory Committee on Immunization Practices Recommended Immunization Schedules for Persons Aged 0 Through 18 Years — United States, 2016
}

\author{
Candice L. Robinson, $\mathrm{MD}^{1}$; Advisory Committee on Immunization Practices (ACIP), ACIP Child/Adolescent Immunization Work Group ${ }^{2}$
}

On February 2, 2016, this report was posted as an MMWR Early Release on the MMWR website (http://www.cdc.gov/mmwr).

Each year, the Advisory Committee on Immunization Practices (ACIP)* reviews the recommended immunization schedules for persons aged 0 through 18 years to ensure that the schedules reflect current recommendations for Food and Drug Administration-licensed vaccines. In October 2015, ACIP approved the recommended immunization schedules for persons aged 0 through 18 years for 2016; the 2016 schedules include several changes from the 2015 immunization schedules. For 2016, the figures, footnotes, and tables will be published on the CDC immunization schedule website (http://www.cdc.gov/ vaccines/schedules/index.html). This provides readers electronic access to the most current version of the schedules and footnotes on the CDC website. Health care providers are advised to use figures, tables, and the combined footnotes together. Printable versions of the 2016 immunization schedules for persons aged 0 through 18 years in several formats (e.g., portrait, landscape, and pocket-sized versions) and ordering instructions for laminated versions and "parent-friendly" schedules are available at the immunization schedule website.

\footnotetext{
${ }^{*}$ http://www.cdc.gov/vaccines/acip/committee/members-archive.html.
}

Recommendations for routine use of vaccines in children, adolescents, and adults are developed by the Advisory Committee on Immunization Practices (ACIP). ACIP is chartered as a federal advisory committee to provide expert external advice and guidance to the Director of the Centers for Disease Control and Prevention (CDC) on use of vaccines and related agents for the control of vaccine-preventable diseases in the civilian population of the United States. Recommendations for routine use of vaccines in children and adolescents are harmonized to the greatest extent possible with recommendations made by the American Academy of Pediatrics (AAP), the American Academy of Family Physicians (AAFP), and the American College of Obstetricians and Gynecologists (ACOG). Recommendations for routine use of vaccines in adults are harmonized with recommendations of AAFP, ACOG, the American College of Physicians (ACP), and the American College of Nurse-Midwives (ACNM). ACIP recommendations adopted by the CDC Director become agency guidelines on the date published in the Morbidity and Mortality Weekly Report (MMWR). Additional information regarding ACIP is available at http://www.cdc.gov/vaccines/acip.
For further guidance on the use of each vaccine included in the schedules, including contraindications and precautions, health care providers are referred to the respective ACIP vaccine recommendations (http://www.cdc.gov/vaccines/hcp/ acip-recs). Providers should be aware that changes in recommendations for specific vaccines can occur between annual updates to the childhood/adolescent immunization schedules.

These immunization schedules are approved by ACIP (http:// www.cdc.gov/vaccines/acip/index.html), the American Academy of Pediatrics (https://www.aap.org), the American Academy of Family Physicians (http://www.aafp.org), and the American College of Obstetricians and Gynecologists (http://www.acog.org).

The most current immunization schedules can be found on the Vaccines and Immunizations pages of CDC's website (http://www.cdc.gov/vaccines/schedules). If errors or omissions are discovered, CDC posts revised versions on these web pages.

$\mathrm{CDC}$ encourages organizations that previously have relied on copying the schedules on their websites to instead use syndication, as a more reliable method for displaying the most current and accurate immunization schedules on an organization's website. Use of content syndication requires a one-time step that ensures an organization's website displays current schedules as soon as they are published or revised; instructions for the syndication code are available on CDC's website (http://www. cdc.gov/vaccines/schedules/syndicate.html). CDC also offers technical assistance for implementing this form of content syndication (e-mail request to ncirdwebteam@cdc.gov).

Changes to the 2016 figures from the previous schedules ${ }^{\dagger}$ are as follows:

- In Figure 1, "Recommended Immunization Schedule for Persons Aged 0 through 18 Years," the order of the vaccines was changed to group vaccines by the recommended age of administration. The order was also changed within the footnotes.

- A purple bar was added for Haemophilus influenzae type b (Hib) vaccine for children aged 5-18 years, denoting the recommendation to vaccinate certain children at high risk in this age group who are unimmunized.

- A purple bar was added for human papillomavirus (HPV) vaccine for children aged 9-10 years, denoting the recommendation to vaccinate children at high risk in this age group, including children with a history of sexual abuse.

\footnotetext{
${ }^{\dagger}$ http://www.cdc.gov/vaccines/schedules/past.html.
} 
- A new row was added for Meningococcal B vaccine. This row contains a purple bar denoting the recommendation to vaccinate certain persons at high risk aged 10 years and older. This row also contains a blue bar denoting the recommendation for administration to groups not at high risk (subject to individual clinical decision making) for persons aged 16 through 23 years (the preferred age range is $16-18$ years).

- In Figure 2, "Catch-up immunization schedule for persons aged 4 months through 18 years who start late or who are more than 1 month behind," Tdap/Td was added to the list of possible previous vaccines in the Tdap line for children aged 7 years and older, dose 2 to dose 3 column.

Changes to the 2016 footnotes from the previous schedules are as follows:

- The Hepatitis B (HepB) vaccine footnote was revised to 1) more clearly present the timing for post-vaccination serologic testing for infants born to mothers whose test results were positive for hepatitis $\mathrm{B}$ surface antigen ( $\mathrm{HBsAg}$ ); and 2) present the new CDC-recommended interval for post-vaccination serologic testing in this population.

- The diphtheria and tetanus toxoids and acellular pertussis (DTaP) vaccine footnote was revised to more clearly present recommendations following an inadvertently early administered 4th dose of DTaP vaccine.
- The inactivated polio vaccine (IPV) footnote was updated to provide guidance for vaccination of persons who received only oral poliovirus vaccine (OPV) and received all doses before age 4 years.

- The meningococcal vaccines footnote was updated to include recommendations for the administration of the meningococcal B vaccine. A "clinical discretion" category was added for the recommendation for vaccination of persons not at high risk aged 16 through 23 years, subject to individual clinical decision making. Meningococcal B vaccines have been added to the section recommending vaccination of persons with high-risk conditions and other persons at increased risk for disease. A definition of persistent complement deficiency has been added.

- The human papillomavirus (HPV) vaccine footnote was updated to reflect the new HPV vaccine nomenclature. Guidance was added for vaccination beginning at age 9 years for children with a history of sexual abuse.

\footnotetext{
${ }^{1}$ Immunization Services Division, National Center for Immunization and Respiratory Diseases, CDC; ${ }^{2}$ Advisory Committee on Immunization Practices (ACIP), ACIP Child/Adolescent Immunization Work Group.

Corresponding author: Candice L. Robinson, CRobinson4@cdc.gov, 404-718-1400.
}

\section{ACIP Child/Adolescent Immunization Work Group}

Work Group chair: Jose Romero, MD, Little Rock, Arkansas.

Work Group members: Allison Kempe, MD, Aurora, Colorado; Cynthia Pellegrini, Washington, DC; H. Cody Meissner, MD, Boston, Massachusetts; Amy B. Middleman, Oklahoma City, Oklahoma; Susan Lett, MD, Boston, Massachusetts; Diane Peterson, Saint Paul, Minnesota; Katie Brewer, MSN, Silver Spring, Maryland; Patricia Stinchfield, MPH, Saint Paul, Minnesota; Rosemary Spence, MA, Denver, Colorado; William L. Atkinson, MD, Harrisonville, Missouri; Jennie Yoost, MD, Huntington, West Virginia; Robin Liu, MD, Portland, Oregon; Don Solimini, MHA, Fishersville, Virginia; Tina Simpson, MD, Birmingham, Alabama.

Work Group Contributors: Andrew Kroger, MD, Atlanta, Georgia; Charles Wolfe, Atlanta, Georgia; Donna Weaver, MN, Atlanta, Georgia; JoEllen Wolicki, Atlanta, Georgia; Akiko Wilson, Atlanta, Georgia; Jennifer Hamborsky, MPH, Atlanta, Georgia; Suzanne Johnson-DeLeon, MPH, Atlanta, Georgia; Raymond Strikas, MD, Atlanta, Georgia. 